\title{
Prevalência de fatores de risco cardiovascular em idosos não institucionalizados
}

\author{
Prevalence of cardiovascular risk factors in non-institutionalized elderly
}

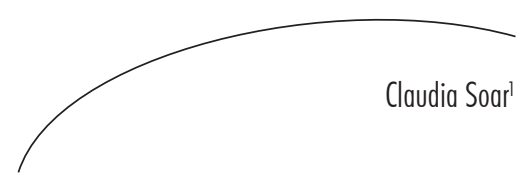

\section{Resumo}

Introdução: $\mathrm{Na}$ medida em que mais pessoas vivem até a idade avançada, aumenta a prevalência de doenças em que a idade é fator de risco, como as doenças cardiovasculares. Objetivo: Descrever as prevalências de fatores de risco para doenças cardiovasculares em idosos não institucionalizados, conforme sexo e grupo etário. Método: Estudo transversal com amostra de conveniência composta por idosos não institucionalizados. Foram analisadas as variáveis hipertensão arterial, hipercolesterolemia, excesso de peso e adiposidade abdominal. Utilizou-se o teste Qui-quadrado para a análise das diferenças estatísticas. Resultados: As prevalências de hipertensão arterial, hipercolesterolemia, excesso de peso e adiposidade abdominal foram, respectivamente, de 82,30\% para idosas e $76,81 \%$ para idosos $(p>0,05), 43,64 \%$ para idosas e $25,48 \%$ para idosos $(p<0,05), 47,40 \%$ para idosas e $36,12 \%$ para idosos $(p<0,05)$ e de $76,16 \%$ para idosas e $41,06 \%$ para idosos $(p<0,05)$. A respeito das diferenças por grupo etário, identificouse menor prevalência, somente para o excesso de peso, à medida que a idade avança. Conclusão: As idosas podem ser consideradas como grupo mais vulnerável para fatores de risco cardiovascular, pois tiveram maior número de medidas com valores acima do recomendado, bem como maior prevalência de hipertensão arterial, hipercolesterolemia, excesso de peso e adiposidade. Com relação ao grupo etário, identificou-se menor prevalência somente para o excesso de peso, à medida que a idade avança.

\section{Abstract}

Introduction: As people live longer, prevalence of diseases in which age is risk factor increase, as cardiovascular disease Objective: To investigate the prevalence of risk factors for cardiovascular diseases in non-institutionalized elderly by different age and sex groups. Method: Cross-sectional study with a convenience sample of non-

\footnotetext{
Universidade do Vale do Paraíba, Faculdade de Ciências da Saúde, Curso de Nutrição. São José dos Campos, SP, Brasil.

Financiamento: Fundação de Amparo à Pesquisa do Estado de São Paulo - FAPESP, processo $\mathrm{n}^{\mathrm{o}}$ 05/55069-9, auxílio à pesquisa.

Palavras-chave: Idoso. Hipertensão. Hipercolesterolemia. Índice de Massa Corporal. Circunferência de Cintura. 
institutionalized elderly. The variables hypertension, hypercholesterolemia, overweight and abdominal adiposity were investigated. The chi-square test was used to analyze statistical differences. Results: The prevalence of hypertension, hypercholesterolemia, overweight and abdominal fat were, respectively, $82.30 \%$ for woman and 76.81 for men ( $p>0.05), 43.64 \%$ for woman and $25.48 \%$ for men $(p<0.05), 47.40 \%$ for woman and $36.12 \%$ for men $(p<0.05)$ and $76.16 \%$ for woman and $41.06 \%$ for men $(p<0.05)$. Prevalence of overweight was lowest in the age group $>80$ year. Conclusion: Older woman may be considered as the most vulnerable group for cardiovascular disease, with variables above recommended, and higher prevalence of arterial hypertension, hypercholesterolemia, overweight and abdominal adiposity. Regarding age group, we identified prevalence in overweight only, as age advances.
Key words: Elderly.

Hypertension.

Hypercholesterolemia.

Body Mass Index. Waist

Circumference.

\section{INTRODUÇÃO}

As doenças crônicas não transmissíveis (DCNT), como as cardiovasculares (DCV), por exemplo, são as principais causas de morte no mundo, configurando-se como o problema de saúde pública de maior magnitude, inclusive no Brasil. ${ }^{1}$

Um dos fatores de risco para as DCVs é o avanço da idade, tornando os idosos um grupo vulnerável para seu surgimento..$^{2-5} \mathrm{O}$ Brasil destaca-se por apresentar uma das maiores taxas de crescimento para população idosa, o que acarreta aumento da carga de doenças, em especial as DCNTs. Teremos uma das maiores populações de idosos do mundo, ocupando as primeiras posições no ranking dos países com maior número de idosos. ${ }^{1,2}$

A elevada prevalência de fatores de risco cardiovascular (FRCV) em idosos, como a presença de hipertensão arterial, obesidade, adiposidade central e dislipidemias, é evidenciada em diversos estudos realizados no Brasil. ${ }^{6-15}$

Dada a relevância da situação e como forma de auxiliar no planejamento para a assistência do grupo populacional que mais cresce no mundo, o objetivo deste estudo foi descrever as prevalências de fatores de risco para doenças cardiovasculares em idosos não institucionalizados, conforme sexo e grupo etário.

\section{MÉTODO}

O estudo teve um delineamento transversal, com amostra por conveniência, com coleta de dados primários de idosos vivendo em comunidade, frequentadores do Instituto Paulista de Geriatria e Gerontologia (IPGG) José Ermírio de Morais, localizado no bairro de São Miguel Paulista, São Paulo-SP, região leste da cidade de São Paulo. O IPGG, antigo Centro de Referência do Idoso (CRI), é o primeiro equipamento de saúde voltado à assistência à saúde da pessoa idosa, mantido pela Secretaria de Estado da Saúde de São Paulo. O período da coleta de dados ocorreu entre 2006 e 2007.

A amostra foi composta por idosos que frequentavam o IPGG, independentemente do tipo de assistência que recebiam. Compuseram a amostra idosos que estavam em atendimento ambulatorial, bem como aqueles que participavam de atividades esportivas ou culturais (aulas de artesanato, dança, ginástica, computação, dentre outras). No momento da coleta, não apresentaram quadro de inflamação e sepse ou diagnóstico de insuficiência cardíaca crônica (ICC), insuficiência renal crônica (IRC), insuficiência hepática (IH) ou qualquer doença que levasse à presença de edema ou desidratação, pois essas condições poderiam alterar as medidas corporais.

O protocolo da pesquisa foi submetido e aprovado pelo Comitê de Ética em Pesquisa da 
Faculdade de Saúde Pública da Universidade de São Paulo, ofício no COEP/64/05. Todos os participantes da pesquisa assinaram o Termo de Consentimento Livre e Esclarecido (TCLE). Os valores de referência, bem como os procedimentos para a coleta das variáveis do estudo seguiram diretrizes e recomendações vigentes no período de aprovação do projeto, ocorrido em 2005.

\section{Foram considerados como FRCV:}

1) Hipertensão arterial (HA) - identificada quando a pressão arterial sistólica (PAS) foi $\geq 140$ $\mathrm{mmHg}$ e/ou a pressão arterial diastólica (PAD) $\geq 90 \mathrm{mmHg}$ e/ou uso contínuo de medicação anti-hipertensiva. A aferição da pressão arterial foi realizada em duplicata, com intervalo de um a dois minutos entre as medidas, pelo método auscultatório, com esfigmomanômetro de coluna de mercúrio. Utilizou-se a média aritmética das medidas. Antes da coleta, o idoso permanecia em descanso por 5-10 minutos. Foi utilizado o "Procedimento de medida da pressão arterial" descrito nas "IV Diretrizes Brasileiras de Hipertensão Arterial" (SBH/SBC/SBN 2002). ${ }^{16}$

2) Hipercolesterolemia (HC) - na presença de colesterol total (CT) sanguíneo $\geq 240 \mathrm{mg}$ / dL e/ou uso contínuo de medicação antihipercolesterolêmica. A coleta da amostra sanguínea, relativa ao exame de CT, ocorreu em até uma semana após a coleta dos demais dados. Os idosos foram orientados a permanecer em jejum de 12 horas. As análises e emissão de laudos das amostras de sangue foram realizadas por laboratório de análises clínicas conveniado da Secretaria de Saúde do Estado de São Paulo, que segue protocolos técnicos de rotina que atendem às normas de biossegurança vigentes pela Sociedade Brasileira de Patologia Clínica. Empregou-se o protocolo para verificação de perfil lipídico da Sociedade Brasileira de Cardiologia (2001) - III Diretrizes Brasileiras sobre Dislipidemias e Diretrizes de Prevenção da Aterosclerose. ${ }^{17}$
3) Excesso de peso - quando o idoso apresentava índice de massa corporal (IMC) $\geq 28 \mathrm{~kg} / \mathrm{m}^{2}$. O IMC foi calculado pela divisão do peso, em quilos, pela estatura, em metros, ao quadrado [IMC $=$ peso $(\mathrm{kg}) \div$ estatura $\left.^{2}(\mathrm{~m})\right]$. Para mensuração de peso, utilizou-se balança eletrônica, da marca Plenna, com capacidade para $150 \mathrm{~kg}$ e precisão de $100 \mathrm{~g}$. Os idosos foram pesados descalços e com roupas leves, posicionados no centro da balança. Para a estatura, utilizou-se fita inextensível com precisão de $0,1 \mathrm{~cm}$, afixada na parede. Os idosos foram posicionados de costas para a parede, descalços, em posição ereta (plano horizontal de Frankfurt). A medida foi obtida quando pelo menos três, dos seguintes pontos: cabeça, ombros, nádegas, panturrilha e calcanhares, estavam encostados na parede. Utilizou-se a recomendação da Organização Pan-americana da Saúde (OPAS). ${ }^{18}$

4) Adiposidade abdominal - quando a circunferência de cintura (CC) fosse $\geq 102 \mathrm{~cm}$, no caso de homens, e $\geq 88 \mathrm{~cm}$, no caso de mulheres, conforme recomendação de Han et al. ${ }^{19}$ Para aferir a CC, o local da medida foi na região do ponto médio entre a crista ilíaca e a última costela, utilizando-se também fita inextensível.

A coleta dos dados antropométricos foi realizada pela pesquisadora, capacitada em treinamento no Laboratório de Avaliação Nutricional de Populações (LANPOP) do Departamento de Nutrição da Faculdade de Saúde Pública da Universidade de São Paulo. As medidas corporais foram obtidas em duplicata, utilizando-se a média aritmética e respeitando o limite de variabilidade entre as medidas, dois milímetros para a estatura e um centímetro para a circunferência de cintura.

Foram avaliados PAS, PAD, CT, IMC e CC, conforme sexo e grupo etário. Os grupos etários foram descritos conforme o Expert Committee da Organização Mundial da Saúde, ${ }^{20}$ que recomenda, para idosos, três grupos com intervalos de dez anos: 60 a 69 anos, 70 a 79 anos, e superior a 80 anos. As medidas foram apresentadas em 
valores médios e intervalos de confiança (IC) de $95 \%$. As diferenças significantes, entre sexo e os grupos etários, foram verificadas pela não interseção do IC.

Foram analisadas as prevalências dos FRCVs, conforme sexo e grupos etários, de HA, HC, excesso de peso e adiposidade abdominal. Para a verificação de diferença significante entre sexo e grupos etários, utilizou-se o teste Quiquadrado com nível de significância de 5\%. Foram utilizadas análises brutas, não ajustadas para possíveis confundidores. Foi utilizado o programa STATA - Statistics/Data Analysis versão 9.2.

\section{RESULTADOS}

Na tabela 1, descreve-se a distribuição da amostra, composta por 955 idosos, segundo grupo etário e sexo. Observou-se distribuição decrescente nas proporções de idosos, à medida que a idade aumenta, tanto no sexo masculino, quanto no feminino. Observou-se também predomínio de mulheres.

Tabela 1. Distribuição de idosos, segundo grupo etário e sexo. São Miguel Paulista-SP, 2008.

\begin{tabular}{lccc}
\hline \multicolumn{1}{c}{ Grupo etário (anos) } & \multicolumn{2}{c}{ Sexo } & Total \\
\hline & Masculino & Feminino & \\
$601-70$ & $148(56,27 \%)$ & $415(59,97 \%)$ & $563(58,95 \%)$ \\
$701-80$ & $101(38,40 \%)$ & $232(33,53 \%)$ & $333(34,87 \%)$ \\
$\geq 80$ & $14(6,18 \%)$ & $45(6,50 \%)$ & $59(6,18 \%)$ \\
Total & $263(100 \%)$ & $692(100 \%)$ & $955(100 \%)$ \\
\hline
\end{tabular}

$\mathrm{Na}$ tabela 2, são apresentados os valores médios e IC $(95 \%)$ de pressão arterial (PAS e PAD), CT, IMC e CC. As mulheres apresentaram valor médio maior de CT e de IMC. Os homens apresentaram valor médio maior de CC.

Tabela 2. Valores médios e intervalos de confiança (IC 95\%) de pressão arterial (PAS e PAD), CT, IMC e CC, conforme sexo. São Miguel Paulista-SP, 2008.

\begin{tabular}{cccccc}
\hline Sexo & $\begin{array}{c}\text { PAS } \\
(\mathrm{mmHg})\end{array}$ & $\begin{array}{c}\text { PAD } \\
(\mathrm{mmHg})\end{array}$ & $\begin{array}{c}\mathrm{CT} \\
(\mathrm{mg} / \mathrm{dL})\end{array}$ & $\begin{array}{c}\mathrm{IMC} \\
\left(\mathrm{kg} / \mathrm{m}^{2}\right)\end{array}$ & $\begin{array}{c}\mathrm{CC} \\
(\mathrm{cm})\end{array}$ \\
\hline Masculino & 135,89 & 82,74 & 202,55 & 26,74 & $99,53^{*}$ \\
& $(133,66-138,12)$ & $(81,56-83,93)$ & $(197,93-207,18)$ & $(26,22-27,25)$ & $(98,18-100,89)$ \\
\hline Feminino & 136,20 & 82,40 & $219,69 *$ & $28,14^{*}$ & 96,13 \\
& $(134,77-137,63)$ & $(81,60-83,19)$ & $(216,48-222,92)$ & $(27,75-28-53)$ & $(95,26-97,00)$ \\
\hline \multirow{2}{*}{ Todos } & 136,11 & 82,49 & 214,97 & 27,75 & 97,07 \\
& $(134,91-137,32)$ & $(81,84-83,16)$ & $(212,28-217,67)$ & $(27,44-28,07)$ & $(96,33-97,81)$ \\
\hline
\end{tabular}

*Os intervalos de confiança não apresentam interseção. 
Em homens, não foi encontrada interseção entre os ICs, de pressão arterial (PAS e PAD), CT, IMC e CC, nos diferentes grupos etários (tabela 3).
Em mulheres observa-se, na tabela 4, tendência à redução dos valores médios entre o grupo etário de 60 anos e o grupo de $\geq 80$ anos. Entretanto, ocorre diferença significativa somente para a variável IMC.

Tabela 3. Valores médios e intervalos de confiança (IC 95\%) de pressão arterial (PAS e PAD), CT, IMC e CC em homens, conforme grupo etário. São Miguel Paulista-SP, 2008.

\begin{tabular}{lccccc}
\hline $\begin{array}{c}\text { Grupo etário } \\
\text { (anos) }\end{array}$ & $\begin{array}{c}\text { PAS } \\
(\mathrm{mmHg})\end{array}$ & $\begin{array}{c}\mathrm{PAD} \\
(\mathrm{mmHg})\end{array}$ & $\begin{array}{c}\mathrm{CT} \\
(\mathrm{mg} / \mathrm{dL})\end{array}$ & $\begin{array}{c}\mathrm{IMC} \\
\left(\mathrm{kg} / \mathrm{m}^{2}\right)\end{array}$ & $\begin{array}{c}\mathrm{CC} \\
(\mathrm{cm})\end{array}$ \\
\hline $601-70$ & 138,15 & 84,20 & 204,86 & 26,77 & 99,12 \\
& $(135,27-141,00)$ & $(82,65-85,75)$ & $(198,36-211,35)$ & $(26,08-27,46)$ & $(97,25-100,98)$ \\
\hline $701-80$ & 133,42 & 81,48 & 202,14 & 27,00 & 100,79 \\
& $(129,67-137,17)$ & $(79,57-83,40)$ & $(195,29-208,98)$ & $(26,16-27,84)$ & $(98,76-102,82)$ \\
\hline$\geq 80$ & 130,00 & 76,43 & 181,21 & 24,48 & 94,91 \\
& $(118,90-141,09)$ & $(70,61-82,25)$ & $(159,58-202,85)$ & $(22,42-26,54)$ & $(87,73-102,09)$ \\
\hline
\end{tabular}

Tabela 4. Valores médios e intervalos de confiança (IC 95\%) de pressão arterial (PAS e PAD), CT, IMC e CC em mulheres, conforme grupo etário. São Miguel Paulista-SP, 2008.

\begin{tabular}{cccccc}
\hline $\begin{array}{c}\text { Grupo etário } \\
\text { (anos) }\end{array}$ & $\begin{array}{c}\text { PAS } \\
(\mathrm{mmHg})\end{array}$ & $\begin{array}{c}\text { PAD } \\
(\mathrm{mmHg})\end{array}$ & $\begin{array}{c}\mathrm{CT} \\
(\mathrm{mg} / \mathrm{dL})\end{array}$ & $\begin{array}{c}\mathrm{IMC} \\
\left(\mathrm{kg} / \mathrm{m}^{2}\right)\end{array}$ & $\begin{array}{c}\mathrm{CC} \\
(\mathrm{cm})\end{array}$ \\
\hline $601-70$ & 135,53 & 82,78 & 219,38 & 28,51 & 96,28 \\
& $(133,63-137,42)$ & $(81,69-83,87)$ & $(215,29-223,46)$ & $(28,00-29,02)$ & $(95,14-97,43)$ \\
\hline $701-80$ & 137,76 & 82,37 & 222,50 & 27,93 & 96,45 \\
& $(135,38-140,16)$ & $(81,13-83,61)$ & $(216,73-228,26)$ & $(27,27-28,58)$ & $(94,95-97,95)$ \\
\hline$\geq 80$ & 134,33 & 79,06 & 208,00 & $25,83 *$ & 93,12 \\
& $(129,19-139,47)$ & $(76,44-81,69)$ & $(195,60-220,80)$ & $(24,44-27,21)$ & $(89,91-96,34)$ \\
\hline
\end{tabular}

*Os intervalos de confiança não apresentam interseção. 
O fator de risco cardiovascular HA esteve presente em cerca de $80 \%$ dos idosos investigados (figura 1); em mulheres, $82,30 \%$; e em homens, $76,81 \%$ (Qui-quadrado $=3,81 ; \quad p>0,05) . \quad \mathrm{Em}$ homens, a prevalência passou de $73,81 \%$ no grupo etário de 60 anos, para $92,86 \%$ em idosos $\geq 80$ anos. Em mulheres, as prevalências se mantiveram em torno de $80 \%$. Não foram encontradas diferenças significativas nos diferentes grupos etários, embora se observasse tendência ao aumento da prevalência de HA, tanto em homens como em mulheres, com o aumento da idade.

Para HC (figura 1), constatou-se maior prevalência $(43,64 \%)$ em mulheres do que em homens (25,48\%) (Qui-quadrado= 26,52; $p<0,00)$. Houve redução na prevalência de $\mathrm{HC}$ em homens com 70 anos e o inverso nas mulheres, mas não foram identificadas diferenças significantes nessas situações.

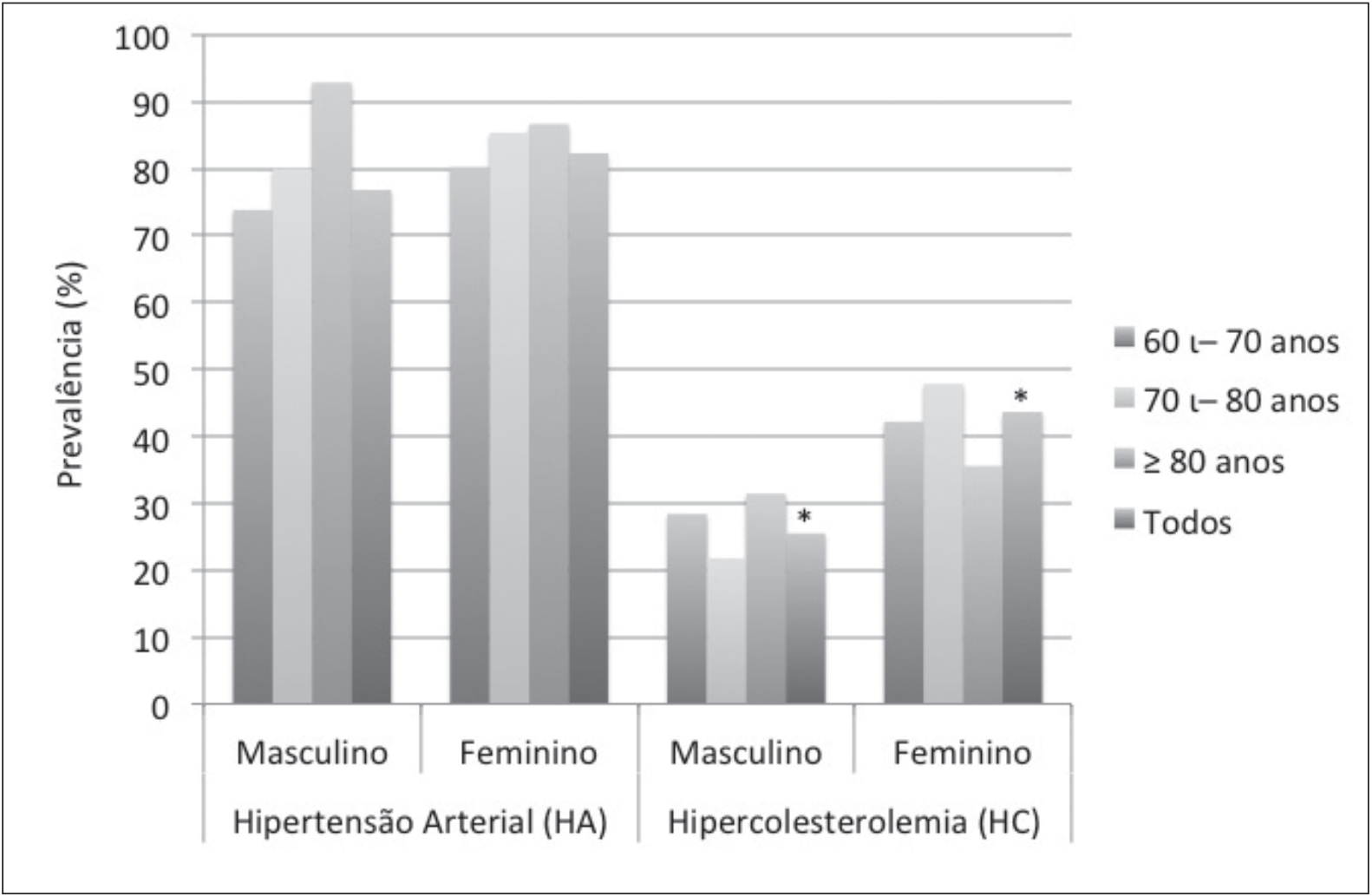

Figura 1. Prevalência dos fatores de risco cardiovasculares hipertensão arterial e hipercolesterolemia, conforme sexo e grupo etário. São Miguel Paulista-SP, 2008.

Foi encontrada diferença significativa no FRCV "excesso de peso" entre os sexos, ocorrendo maior prevalência em mulheres $(47,40 \%) \quad$ (Qui-quadrado $=9,82 ; \quad p=0,00)$, do que em homens $(36,12 \%)$. Ocorreu redução na prevalência à medida que a idade aumenta.
Observa-se prevalência de adiposidade abdominal estaticamente maior em mulheres (76,16\%) (Quiquadrado $=105,33 ; p=0,00)$ do que nos homens $(41,06 \%)$. Em homens, as prevalências apresentam tendência à redução com o aumento da idade, porém sem diferença significativa (figura 2). 


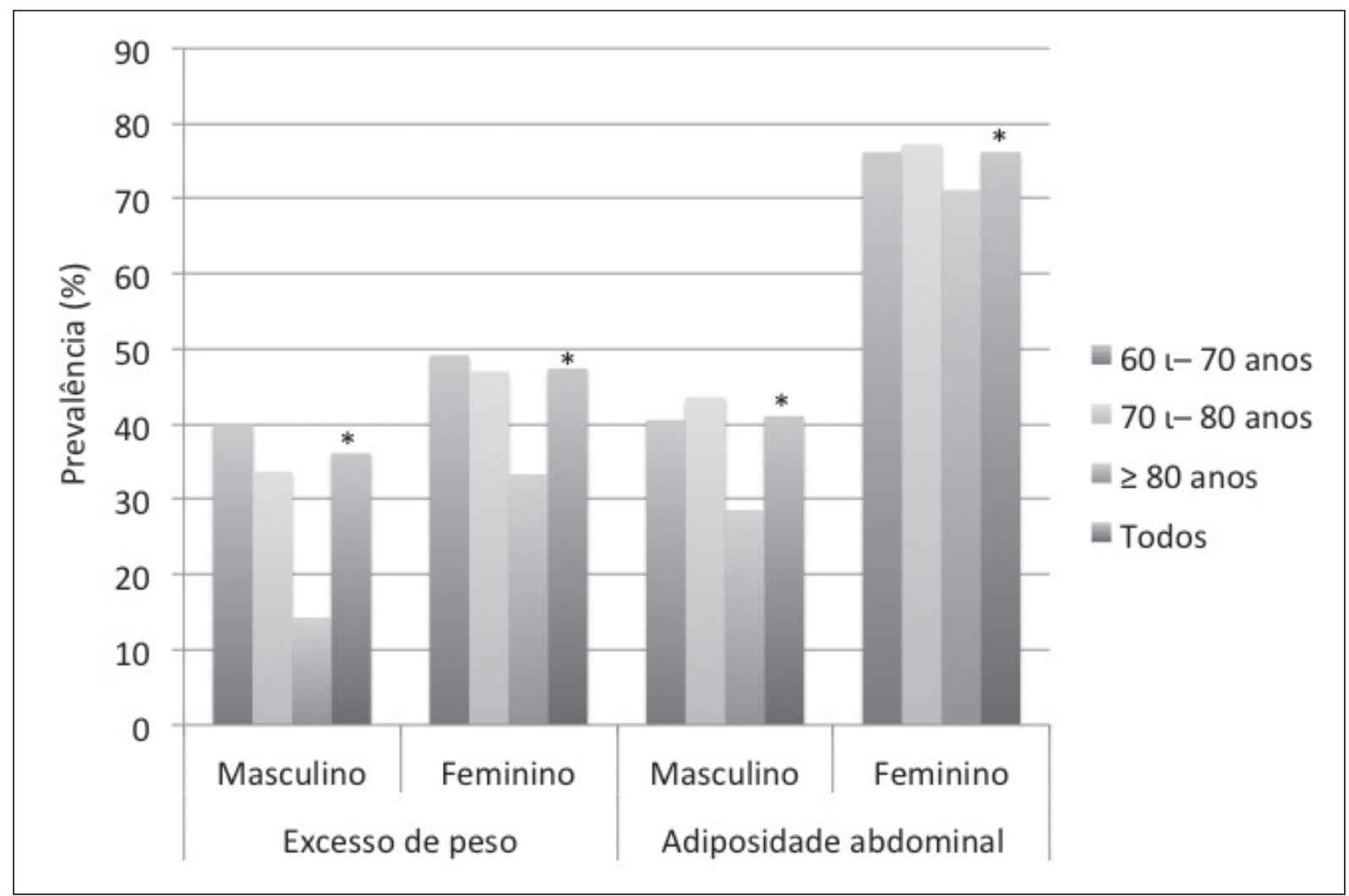

Figura 2. Prevalência dos fatores de risco cardiovasculares excesso de peso e adiposidade abdominal, conforme sexo e grupo etário. São Miguel Paulista-SP, 2008.

\section{DISCUSSÃO}

Os resultados de proporções de idosos por grupo etário identificados no estudo vão ao encontro de dados publicados anteriormente. , $^{3,21,22}$ O predomínio de mulheres, em praticamente todas as faixas etárias, e que se acentua com o aumento da idade, vem sendo observado no Brasil em diversos estudos de base populacional, como a Pesquisa Nacional sobre Saúde e Nutrição $(\mathrm{PNSN})^{23}$ e a Pesquisa Nacional por Amostras de Domicílios (PNAD), ${ }^{24}$ bem como em estudos específicos da população idosa, como os resultados descritos por Cabrera \& Jacob Filho, ${ }^{7}$ Menéndez et al., ${ }^{25}$ Santos \& Sichieri, ${ }^{26}$ Bassler \& Lei $^{27}$ e Silveira et al. ${ }^{28}$ Tal achado replica-se globalmente, possivelmente por mortalidade mais precoce entre os homens, resultando em maior expectativa de vida para as mulheres.
Também seguindo a tendência mundial, o predomínio de uma população idosa jovem, no grupo etário entre 60 e 70 anos, também foi encontrada neste estudo. Esse fato, certamente, influencia o perfil de saúde, visto que pessoas muito idosas são geralmente mais frágeis e demandam serviços de maior complexidade. ${ }^{29} \mathrm{O}$ número menor de idosos com mais de 80 anos pode ter sido influenciado pela característica da amostra. Os idosos que frequentam o IPGG são aqueles com maior facilidade de locomoção e melhor condição cognitiva, condições mais comuns em idosos mais jovens.

Em relação aos valores de pressão arterial, observaram-se valores semelhantes em homens e mulheres, como também encontrado por Van Rossum et al. ${ }^{30}$ Já Barreto et al. ${ }^{8}$ apresentaram valores médios de PAS e PAD maiores em 
homens, e Cruz et al. ${ }^{9}$ verificaram valores médios maiores em mulheres. Observou-se tendência à diminuição dos valores de pressão em homens, tanto sistólica como diastólica, que foi contrária aos estudos de Van Rossum et al. ${ }^{30}$ e Barreto et al., ${ }^{8}$ nos quais os valores de PAS e PAD em idosos aumentavam com o avanço da idade.

Foram encontradas prevalências de HA maiores do que as descritas para a população adulta, bem como as descritas em estudos específicos com população idosa. Em publicação oficial do Ministério da Saúde (Cadernos de Atenção Básica - Hipertensão Arterial Sistêmica), ${ }^{31}$ apontou-se que, no Brasil, a prevalência varia entre 22 e $44 \%$ para adultos, chegando a mais de $50 \%$ para indivíduos com 60 a 69 anos e $75 \%$ em indivíduos com mais de 70 anos. Recentemente, em estudo de metanálise de base populacional sobre a prevalência de hipertensão em idosos brasileiros, Picon et al. ${ }^{32}$ apontaram prevalência de 68\% no período entre 1980 e 2010. As prevalências apresentadas neste estudo podem ser maiores do que as apresentadas em outros estudos, por conta dos diferentes critérios diagnósticos utilizados. Em muitos estudos, utiliza-se PAS $\geq 160 \mathrm{mmHg}$ e/ou PAD $\geq 95$ $\mathrm{mmHg}$ ou considera-se somente pressão arterial sistólica ou hipertensão autorrelatada.

$\mathrm{Na}$ comparação entre sexos, o resultado encontrado foi semelhante a diversos estudos, ou seja, a HA é mais frequente em mulheres. , $11,19,33-36^{-36}$ Estimativas globais sugerem prevalência de hipertensão mais elevada para homens até os 50 anos de idade e para mulheres a partir da sexta década. ${ }^{37}$

Um dos fatores que pode favorecer esse quadro é que as mulheres geralmente têm maior percepção das doenças, apresentam maior tendência para o autocuidado e buscam mais assistência médica do que os homens, o que tenderia a aumentar a probabilidade de ter a HA diagnosticada. ${ }^{11}$ Com relação às comparações entre grupo etário, embora não se tenham identificado diferenças estatísticas, há tendência ao aumento da prevalência de HA entre os grupos etários, sendo maior no grupo etário mais idoso, semelhante a outros estudos. ${ }^{12,30}$

Observou-se valor médio de CT maior em mulheres do que em homens, o que também foi identificado por Martins et al. ${ }^{13}$ e, possivelmente, possa refletir a maior prevalência de $\mathrm{HC}$ em mulheres. A prevalência de $\mathrm{HC}$, observada no estudo, maior em mulheres do que em homens, também foi identificada por Alencar et al., ${ }^{33}$ embora neste, com prevalência menor. Diferentemente desse resultado, Cabrera \& Jacob Filho ${ }^{7}$ constataram prevalência maior em homens do que em mulheres. Também se identificou, em mulheres, aparente diminuição das prevalências com o aumento da idade, de acordo com estudo de Chan et al. ${ }^{38}$

É importante ressaltar prováveis fatores confundidores nesse achado. Há possibilidade de a idade média inferior no sexo feminino na amostra, possivelmente em função do número grande de mulheres entre 60 e 70 anos. Sabese que a idade mais avançada é preditor de fragilidade, sendo o CT inferior em pacientes frágeis - ressalta-se, assim, a importância de uma análise multivariada para melhor avaliar tal situação.

Em relação ao IMC, os resultados apresentados no estudo assemelham-se a diversos estudos nacionais e internacionais. ${ }^{7,8,26,34,35,39-41}$ Os valores para homens e mulheres estiveram, inclusive, dentro dos valores considerados adequados pela OPAS, ${ }^{18}$ ou seja, entre 23 e $28 \mathrm{~kg} / \mathrm{m}^{2}$. É importante ressaltar que, como em outros estudos, as mulheres apresentaram valor médio de IMC maior do que os homens. ${ }^{7,14,26,34,35,41,42}$ Ainda sobre o IMC, no presente estudo ocorreu decréscimo dos valores médios com o avanço da idade em mulheres, o que também foi identificado em outros estudos. ${ }^{7,26,39,43,44}$

A comparação com outros estudos em relação à prevalência de excesso de peso utilizando o IMC como indicador não foi possível, pois os 
valores aqui adotados, como excesso de peso, somente foram usados no estudo SABE..$^{32}$ Havia, no entanto, uma categorização diferenciada de IMC, considerando excesso de peso na presença de IMC entre 28 e $30 \mathrm{~kg} / \mathrm{m}^{2}$ e obesidade a partir de IMC $30 \mathrm{~kg} / \mathrm{m}^{2}$. Se considerarmos idosos com excesso de peso aqueles com IMC a partir de 28 $\mathrm{kg} / \mathrm{m}^{2}$, no estudo $\mathrm{SABE}^{14}$ a prevalência de excesso de peso encontrada seria de $40,5 \%$ para mulheres e $21,7 \%$ em homens, menor do que a encontrada no presente estudo. Independentemente das categorias de IMC utilizada na identificação de excesso de peso/sobrepeso/obesidade, este estudo assemelha-se a outros trabalhos que apresentam predomínio de mulheres na condição "excesso de peso". 7,14,26,39

O excesso de peso, além de fator de risco independente para o desenvolvimento de DCV, é fator predisponente para a HA, podendo ser responsável por 20 a $30 \%$ dos casos da doença. ${ }^{45}$ Em estudos com idosos, foi evidenciado que o excesso de peso está relacionado ao risco de HA e demais DCV. $7,9,34,46$

Para CC, foi observado que os homens apresentaram valor médio significativamente maior, como verificado em outros estudos. ${ }^{26,34,39}$ Esse resultado está de acordo com a recomendação para adultos, que sugere que, para homens, os valores indicativos de risco de morbidades devem ser maiores. Os valores de CC encontrados em homens e mulheres foram maiores do que os valores descritos na maioria dos estudos que analisaram CC. ${ }^{26,34,39,41}$ Embora não exista recomendação de valor de CC específico para população idosa indicativo de risco para DCV, os valores médios encontrados em homens, em todos os grupos etários, estavam abaixo dos valores descritos para homens adultos como risco nível 2 para DCV. Em mulheres, os valores médios, em todos os grupos etários, estavam acima dos valores descritos para mulheres adultas como risco nível 2 para DCV.

$\mathrm{Na}$ análise conforme grupo etário, observouse tendência, aos maiores valores, no grupo etário de 70 anos, semelhante ao estudo de Perissinotto et al. ${ }^{39}$ Além disso, foram observados valores semelhantes a outros estudos, e também que as mulheres apresentaram maior prevalência de adiposidade abdominal, que usaram o nível 1 de risco de DCV para adultos, ou seja, CC $\geq 94 \mathrm{~cm}$ para homens e $\geq 80 \mathrm{~cm}$ para mulheres, ocorreu predomínio de adiposidade abdominal em mulheres. ${ }^{14,26,40}$

As principais limitações do estudo dizem respeito às características da amostra estudada, obtida por demanda espontânea restrita a idosos vivendo em comunidade, ativos de um centro de referência para idosos e com acompanhamento médico e nutricional frequentes, além de pequeno tamanho amostral da categoria mais 80 anos de idade e análise bruta dos dados. A coleta dos dados antropométricos foi realizada apenas pela pesquisadora, favorecendo a homogeneidade dos dados.

\section{CONCLUSÃO}

A população estudada assemelha-se ao perfil de idosos brasileiros e de outros países no que diz respeito à distribuição etária e sexo, sendo composta por idosos jovens com predomínio de mulheres. As idosas podem ser consideradas como grupo mais vulnerável para fatores de risco cardiovascular, pois tiveram maior número de medidas com valores acima do recomendado, segundo diretrizes e recomendações vigentes no período pesquisado, bem como maior prevalência de hipertensão arterial, hipercolesterolemia, excesso de peso e adiposidade.

Com relação ao grupo etário, identificouse menor prevalência somente para o excesso de peso, à medida que a idade avança. Os resultados aqui obtidos podem ser utilizados como comparativos, bem como auxílio no planejamento de ações que reduzam os fatores de risco cardiovasculares, especialmente na população idosa estudada. 


\section{REFERÊNCIAS}

1. Brasil. Ministério da Saúde, Secretaria de Vigilância em Saúde, Departamento de Análise de Situação de Saúde. Plano de ações estratégicas para o enfrentamento das doenças crônicas não transmissíveis (DCNT) no Brasil 2011-2022. Brasília, DF : Ministério da Saúde; 2011. (Série B. Textos Básicos de Saúde).

2. Brasil. Ministério da Saúde, Secretaria de Atenção à Saúde. Envelhecimento e saúde da pessoa idosa. Brasília, DF: Ministério da Saúde; 2007. (Série A. Normas e manuais técnicos); (Cadernos de atenção básica; no 19).

3. Laurenti R, Jorge MHPM, Gotllieb SLD. Perfil epidemiológico da morbi-mortalidade masculina. Ciênc Saúde Coletiva 2005;10(1):35-46.

4. Tuomilehto J. Impact of age on cardiovascular risk: implications for cardiovascular disease management. J Atheroscler Suppl 2004;5(2):9-17.

5. Hajian-Tilaki KO, Heidari B. Prevalence of obesity, central obesity and the associated factors in urban population aged 20-70 years, in the north of Iran: a population-based study and regression approach. Obes Rev 2007;8(1):3-10.

6. Pinheiro RS, Viacava F, Travassos C, Brito AS. Gênero, morbidade, acesso e utilização de serviços de saúde no Brasil. Ciênc Saúde Coletiva 2002;7(4):687-707.

7. Cabrera MAS, Jacob W Filho. Obesidade em idosos: prevalência, distribuição e associação com hábitos e co-morbidades. Arq Bras Endocrinol Metab 2001;45(5):494-501.

8. Barreto SM, Passos VMA, Lima-Costa MFF. Obesidade e baixo peso entre idosos brasileiros: Projeto Bambuí. Cad Saúde Pública 2003;19(2): 605-12.

9. Cruz IBM, Almeida MSC, Schwanke CHA, Moriguchi EH. Prevalência de obesidade em idosos longevos e sua associação com fatores de risco e morbidades cardiovasculares. Rev Assoc Med Bras 2004;50(2):172-7.

10. Feijão AMM, Gadelha FV, Bezerra AA, Oliveira AM, Silva MSS, Lima JWO. Prevalence of excessive weight and hypertension in a low-income urban population. Arq Bras Cardiol 2005;84(1):29-33.

11. Zaitune MP, Barros MB, Cesar CL, Carandina L, Goldbaum M. Arterial hypertension in the elderly: prevalence, associated factors, and control practices in Campinas, São Paulo, Brazil. Cad Saúde Pública 2006;22(2):285-94.

12. Lebrão ML, Laurenti R. Condições de saúde. In: Lebrão ML, Duarte YAO. SABE - Saúde, Bem-estar e Envelhecimento- O projeto SABE no município de São Paulo: uma abordagem inicial. Brasília, DF: Organização Pan-Americana da Saúde; 2003. p. 75-91.
13. Martins IS, Marucci MFN, Cervato AM, Okani ET, Mazzilli RN, Casajus MI. Doenças cardiovasculares ateroscleróticas, dislipidemias, hipertensão, obesidade e diabetes melito em população da área metropolitana da região Sudeste do Brasil: II - Dislipidemias. Rev Saúde Pública 1996;30(1):75-84.

14. Marucci MFN, Barbosa AR. Estado nutricional e capacidade física. In: Lebrão ML, Duarte YAO. SABE - Saúde, Bem-estar e Envelhecimento - O projeto SABE no município de São Paulo: uma abordagem inicial. Brasília, DF: Organização PanAmericana da Saúde; 2003. p. 93-117.

15. Ferreira CCC, Peixoto MRG, Barbosa MA, Silveira EA. Prevalência de fatores de risco cardiovascular em idosos usuários do Sistema Único de Saúde de Goiânia. Arq Bras Cardiol 2010;95(5):621-8.

16. Sociedade Brasileira de Hipertensão; Sociedade Brasileira de Cardiologia; Sociedade Brasileira de Nefrologia. $4^{a}$ Diretrizes Brasileiras de Hipertensão Arterial. Campos do Jordão: SBH/SBC/SBN; 2002.

17. Sociedade Brasileira de Cardiologia. $3^{\text {a }}$ Diretrizes Brasileiras sobre Dislipidemias e Diretrizes de Prevenção da Aterosclerose do Departamento de Aterosclerose da Sociedade Brasileira de Cardiologia. Arq Bras Cardiol 2001;77sup 3:1-48.

18. Organización Panamericana de La salud. Encuesta Multicêntrica Salud Beinestar y Envejecimiento (SABE) en América Latina y el Caribe: informe preliminar. 36a Reunión Del Comitê Asesor de Investigaciones em Salud; 9-11 de julio de 2001; Jamaica. Washington, DC: División de Promoción y Protección de La Salud (HPP); 2001.

19. Han TS, Leer EV, Seidell C, Lean MEJ. Waist circumference action levels in the identication of cardiovascular risk factors: prevalence study in a random sample. BMJ 1995;311:1401-05.

20. Onis M, Habicht JP. Anthropometric reference data for international use: recommendations from a World Health Organization Expert Committee. Am J Clin Nutr 1996;64(4):650-8

21. Karsch UM. Dependent seniors: families and caregivers. Cad Saúde Pública 2003;19(3):861-6.

22. Pinheiro RS, Viacava F, Travassos C, Brito AS. Gênero, morbidade, acesso e utilização de serviços de saúde no Brasil. Ciênc Saúde Coletiva 2002;7(4):687-707.

23. Instituto Nacional de Alimentação e Nutrição. Pesquisa Nacional sobre Saúde e Nutrição: condições nutricionais da população brasileira: adultos e idosos. Brasília, DF: INAN; 1991. 
24. Instituto Brasileiro de Geografia e Estatística. Pesquisa nacional por amostra de domicílios (PNAD). Rio de Janeiro: IBGE; 1999.

25. Menéndez J, Guevara A, Arcia N, Díaz EMC, Marín C, Alfonso JC. Enfermedades crónicas y limitación funcional en adultos mayores: estudio comparativo en siete ciudades de América Latina y el Caribe. Rev Panam Salud Pública 2005;17(5/6):353-61.

26. Santos DM, Sichieri R. Índice de massa corporal e indicadores antropométricos de adiposidade em idosos. Rev Saúde Pública 2005;39(2):163-8.

27. Bassler TC, Lei DLM. Diagnóstico e monitoramento da situação nutricional da população idosa em município da região metropolitana de Curitiba (PR). Rev Nutr 2008;21(3):311-21.

28. Silveira EA, Kac G, Barbosa LS. Prevalência e fatores associados à obesidade em idosos residentes em Pelotas, Rio Grande do Sul, Brasil: classificação da obesidade segundo dois pontos de corte do índice de massa corporal. Cad Saúde Pública 2012;25(7):1569-77.

29. Instituto Brasileiro de Geografia e Estatística [Internet]. Rio de Janeiro: IBGE; [1995 -]. Tábuas Completas de Mortalidade 2011; 2010 [acesso em 10 de fev 2014]; [aproximadamente 2 telas]. Disponível em: http://www.ibge.gov.br/home/estatistica/ populacao/tabuadevida/2011/default.shtm

30. Van Rossum CTM, Van de Mheen H, Witteman JCM, Hofman A, Mackenbach JP, Grobbee DE. Prevalence, treatment, and control of hypertension by sociodemographic factors among the Dutch elderly. Hipertension 2000;35:814-21.

31. Ministério da Saúde. Estratégia para o cuidado da pessoa com doença crônica/Hipertensão arterial sistêmica. Brasília, DF: Ministério da Saúde; 2013. (Série A Normas e manuais técnicos); (Cadernos de Atenção Básica; no 37).

32. Picon RV, Fuchs FD, Moreira LB, Fuchs SC. Prevalence of hypertension among elderly persons in urban Brazil: a systematic review with meta-analysis. Am J Hypertens 2013;26(4):541-8.

33. Alencar YMG, Carvalho FET, Paschoal SMP, Curiati JAE, Wong CP, Litvoc J. Risk factors for atherosclerosis in an elderly out patient population in the City of São Paulo. Arq Bras Cardiol 2000;74(3):189-96.

34. Dey KD, Rothenberg E, Sundl V, Bosaeus I, Steen B. Waist circumference, body mass index, and risk for stroke in older people. J Am Geriatr Soc 2002;50(9):1510-9.

35. Mirmiran P, Esmaillzadech A, Azizi F. Detection of cardiovascular risk factors by anthropometric measures in Tehranian adults: receiver operating characteristic (ROC) curve analysis. Eur J Clin Nutr 2004;58(8):1110-8.

36. Talleruphuus U, Bang LE, Wiinberg N, Mehlsen J, Svendsen TL, Bentzon MW. Isolated systolic hypertension in an elderly Danish population. Prevalence and daytime ambulatoty blood pressure. Blood Press 2006;15(6):347-53.

37. Sociedade Brasileira de Cardiologia; Sociedade Brasileira de Hipertensão; Sociedade Brasileira de Nefrologia. $5^{a}$ Diretrizes Brasileiras de Hipertensão Arterial.São Paulo: SBC/SBH/SBN; 2006.

38. Chan Y, Suzuki M, Yamamoto S. A comparison of anthropometry, biochemical variables and plasma aminoacids among centenarians, elderly and yong subjects. J Am College Nutr 1999;18(4):358-65.

39. Perissinotto E, Pisent C, Sergi G, Grigoletto F, Enzi G. Anthropometric measurements in the elderly: age and gender differences. Br J Nutr 2002;87:177-86.

40. Gutiérrez-Fisac JL, López E, Banegas JR, Graciani A, Rodríguez-Artalejo F. Prevalence of overweight and obesity in elderly people in Spain. Obes Res 2004;12(4):710-5.

41. Sampaio LR, Figueiredo VC. Correlação entre índice de massa corporal e os indicadores antropométricos de distribuição de gordura corporal em adultos e idosos. Rev Nutr 2005;18(10):53-61.

42. Santos AC, Barros H. Prevalence and determinants of obesity in an urban sample of Portuguese adults. Public Health 2003;117(6):430-37.

43. Burr ML, Phillips KM. Anthropometric norms in the elderly. Br J Nutr 1984;51:165-9.

44. Kuczsmarski MF, Kuczsmarski RJ, Najjar M. Descriptive anthropometric reference data for older Americans. J Am Diet Assoc 2000;100(1):59-66.

45. Wold Health Organization. Obesity: preventing and managing the global epidemic. Report of WHO Consultation on Obesity. Geneva: WHO;1997.

46. Franklin SS, Pio JR, Wong ND, Larson MG, Leip EP, Vasan RS, et al. Predictors of new-onset diastolic and systolic hypertension : the Framingham Heart Study. Circulation 2005;111(9):1121-27. 